\title{
Experimental Neonatal Syphilis. II. Immunological Responses of Neonatal Rabbits to Intradermal Inoculation with Treponema pallidum (Nichols Strain)
}

\author{
DARLENE GAMBOA, JAMES N. MILLER, SHEILA A. LUKEHART,' SHARON A. BAKER- \\ ZANDER,' AND STEWART SELL ${ }^{2}$
}

Treponemal Research Laboratory, Department of Microbiology and Immunology, School of Medicine, University of California at Los Angeles, Los Angeles, California 90024 and Department of Pathology, School of Medicine, University of California at San Diego, La Jolla, California 92093

\section{Summary}

The immunological competence of neonatal rabbits inoculated intradermally with Treponema pallidum was examined. Both cellular responses and the production of humoral antibody to specific $T$. pallidum antigens and to nonspecific antigens or mitogens were investigated. In blast transformation assays, splenic and popliteal lymph node lymphocytes from neonates inoculated with virulent $T$. pallidum responsed to $T$. pallidum antigens in a manner similar to or greater than inoculated adult rabbits. Splenic and popliteal lymph node lymphocytes from both uninoculated and $T$. pallidum-inoculated neonate and adult animals showed consistent and similar responses to concanavalin $\mathbf{A}$. Both neonate and adult animals inoculated with heat-killed $\boldsymbol{T}$. pallidum also responded but to a significantly lesser degree. Immunofluorescent examination of skin sections from the site of inoculation of adult and neonatal animals revealed 1) that the early infiltrate was composed predominntly of $T$ cells, 2) diffuse antibody staining with rare $B$ cells, and 3 ) fewer treponemes with significant fragmentation in neonates as compared to adult controls. Antibody production by neonates inoculated with virulent $T$. pallidum was delayed 4 to 6 weeks postinoculation as measured by the fluorescent Treponemal antibody absorption and Venereal Disease Research Laboratory (VDRL) test procedures, respectively. Antibody was not detected among neonates inoculated with heat-killed treponemes during a 6-week observation period and only low levels of VDRL antibody were detected in a few adult control animals.

Evidence for incomplete resistance of neonatal rabbits to the intradermal inoculation of Treponema pallidum was provided. Twenty-two of 23 neonatal rabbits resistant to symptomatic infection upon initial inoculation with treponemes were also resistant to homologous challenge 3 to 5 months later, thus indicating a refractive state. Additional evidence was provided by the appearance of generalized lesions among seven of 29 neonates.

Received August 1, 1983; accepted March 12, 1984

Address reprint requests to Darlene Gamboa, Department of Biology, California State University at San Bernardino, San Bernardino, CA 92407.

This study was supported by United States Public Health Service Grants AI12601 and AI-19810 from the National Institutes of Health to J. N. M. and S. S., respectively, and by World Health Organization Agreement V3/181/26 to J. N. M. The work was in partial fulfillment of the requirements for the Doctor of Philosophy degree. UCLA, by D. G.

'Present address: Department of Medicine-Infectious Diseases, Seattle Public Health Hospital, 1131 14th Avenue South, Seattle, Washington 98114.

${ }^{2}$ Present address: Department of Pathology and Laboratory Medicine, University of Texas Health Science Center at Houston, Houston, TX 75235.

\section{Abbreviations}

VDRL, Venereal Disease Research Laboratory

FTA-ABS, fluorescent treponemal antibody absorption

Con A, concanavalin A

les(+), lesion positive

ATS, anti-rabbit thymocyte serum

dil, dilutant

In a previous study (9), it was shown that neonatal rabbits exhibit resistance to symptomatic infection with Treponema pallidum as measured by the absence or atypical appearance of lesions following intradermal inoculation. These findings alone, however, do not permit assessment of the status or mechanism of the observed neonatal resistance. Conclusions of this nature may be based at least in part upon data relating to 1) whether their response represents asymptomatic infection or complete elimination of the organisms with or without a local transient reaction, and 2) the immunocompetent state of the inoculated neonates. Several investigators have demonstrated that infected adult rabbits become refractive to reinfection $(3,4,39)$ thereby presenting a means for determining complete versus incomplete resistance among inoculated neonates on the basis of the results of subsequent challenge. The well defined cellular and humoral immune responses of adult rabbits to $T$. pallidum inoculation $(17,18,28,39)$ provide a sound basis for assessing the comparative extent of neonatal immunocompetence.

In the present study, it will be shown that neonatal rabbits inoculated intradermally with $T$. pallidum 1) elicit cellular responses comparable to those of the adult rabbit, 2) show comparative delays in VDRL and FTA-ABS antibody responses, and 3) are resistant to homologous intradermal challenge. Further, the development of generalized lesions among neonates in this study will be demonstrated.

\section{MATERIALS AND METHODS}

Rabbits. Adult ( $\geq 6$ months old) New Zealand albino rabbits with nonreactive VDRL serologic tests were used throughout this study. Neonates were offspring of VDRL nonreactive animals mated in this laboratory; unless otherwise specified, they were housed with their doe until they reached approximately 6 to 8 weeks of age, at which time they were weaned. All rabbits were housed at 18 to $21^{\circ} \mathrm{C}$ and given antibiotic-free food and water ad libitum. 
Source of T. pallidum. T. pallidum, Nichols strain, was maintained by intratesticular passage in rabbits as previously described (9). The nonviability of a control suspension of heat-killed $T$. pallidum $\left(56^{\circ} \mathrm{C}, 1 \mathrm{~h}\right)$ was confirmed as described by Miller et al. (23).

Infection and challenge. Neonates 5 to 8 days of age were inoculated intradermally at two sites on their clipped backs with $0.05 \mathrm{cc}$ of a suspension containing $2 \times 10^{7}$ virulent or heat-killed T. pallidum $/ \mathrm{ml}$ for a total of $1 \times 10^{6}$ treponemes/site. Adult control animals were inoculated in the same manner at two to four sites. All animals were monitored for lesion development; aspirates of representative lesions were examined by dark field microscopy for the presence of motile treponemes.

Groups of animals were sacrificed at 1, 2, 4, and 6 weeks postinoculation for lymphocyte transformation assays. Remaining animals which had received virulent $T$. pallidum were observed for 3 months after which time they were challenged by intradermal inoculation at two to four sites with $1 \times 10^{6}$ treponemes/site.

Lymphocyte transformation assay. Lymphocyte transformation assays were carried out utilizing splenic and popliteal lymph node lymphocytes in the presence of sonicated $T$. pallidum (Nichols) or Con A as described by Lukehart et al. (18). Lymphocyte responsiveness was tested at $1,2,4$, and 6 weeks postinoculation. Adult animals inoculated with heat-killed treponemes were also tested at 12 weeks postinoculation.

Serological testing. The VDRL slide flocculation test and FTAABS test were performed as described in the Manual of Tests for Syphilis (21). Modifications for use with rabbit sera included known positive and negative rabbit control sera and fluoresceinlabeled goat anti-rabbit IgG (Cappel Laboratories, Inc., Davis, CA) used at a final dilution of 1:1280. Titers are expressed as the reciprocal of the highest dilution exhibiting reactive or $2+\mathrm{im}$ munofluorescence for VDRL and FTA-ABS test results, respectively.

Examination of dermal lesions by indirect immunofluorescence. Tissue specimens approximately $1 \times 1 \times 0.3 \mathrm{~cm}$ in size were fixed, stained for immunofluorescence, and examined as described by Lukehart et al. (17) and Sell et al. (32). Human anti-T. pallidum serum, rabbit anti-human globulin, goat antirabbit Ig, goat ATS, and burro anti-goat IgG, either unconjugated or conjugated wth fluorescein isothiocyanate were prepared and employed in the indirect immunofluorescence procedures as previously described $(17,32)$.

Statistics. The geometric mean of replicate cultures for individual animals was determined and unstimulated background values $(50-550)$ subtracted. The mean and standard errors of response from neonate and adult animals at each time point were determined. The geometric mean and standard errors for the reciprocal titers of VDRL and FTA-ABS tests were determined at $1,2,4,6,8$, and 12 weeks postinoculation time points for neonate and adult control animals.

\section{RESULTS}

Lymphocyte responsiveness to T. pallidum antigens. Evidence for the immunocompetency of neonatal rabbits inoculated with virulent $T$. pallidum at age 5 to 8 days was provided by the demonstration of splenic and popliteal lymph node lymphocyte responsiveness to $T$. pallidum antigens at various time points following inoculation. The response of splenic lymphocytes from neonates was lower than that of lymphocytes from similarly infected adults at the initial time point of 1 week postinoculation but subsequently showed similar responses at the 2,4 , and 6 week time points of measurement (Fig. 1). However, popliteal lymph node lymphocytes from neonatal animals exhibited a more pronounced response to antigens than lymphocytes from adults after the initial time point of 2 weeks postinoculation; lymph node lymphocytes from adults exhibited a maximum response of $219 \mathrm{cpm}$ at 2 weeks postinoculation as compared to the minimum neonate response of $930 \mathrm{cpm}$ at the same time

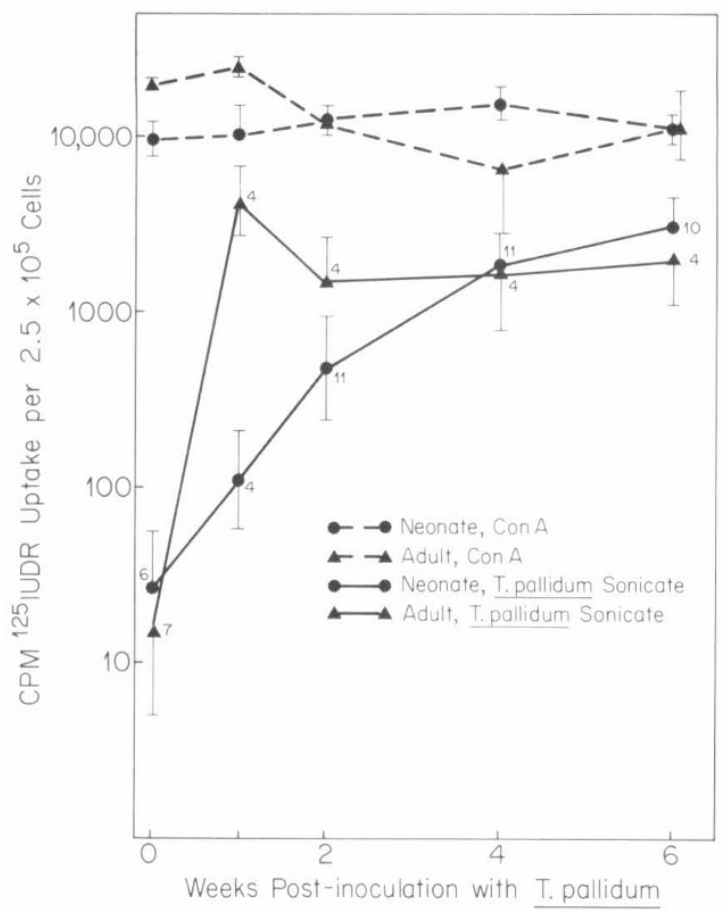

Fig. 1. In vitro proliferative response of splenic lymphocytes from neonatal and adult control rabbits to sonicated $T$. pallidum and Con A after intradermal inoculation with $1 \times 10^{6}$ virulent $T$. pallidum at each of two or four sites. Neonates were inoculated at 5 to 8 days of age. The responses of normal noninfected animals are designated at the 0 time point. The number of animals represented at each time point is indicated. The data points are the geometric mean $\pm \mathrm{SE}$ of $\left[{ }^{125} \mathrm{I}\right]$ iododeoxyuridine $\mathrm{cpm}$ incorporated in six replicate cultures per animal (see Ref. 18 for culture conditions).

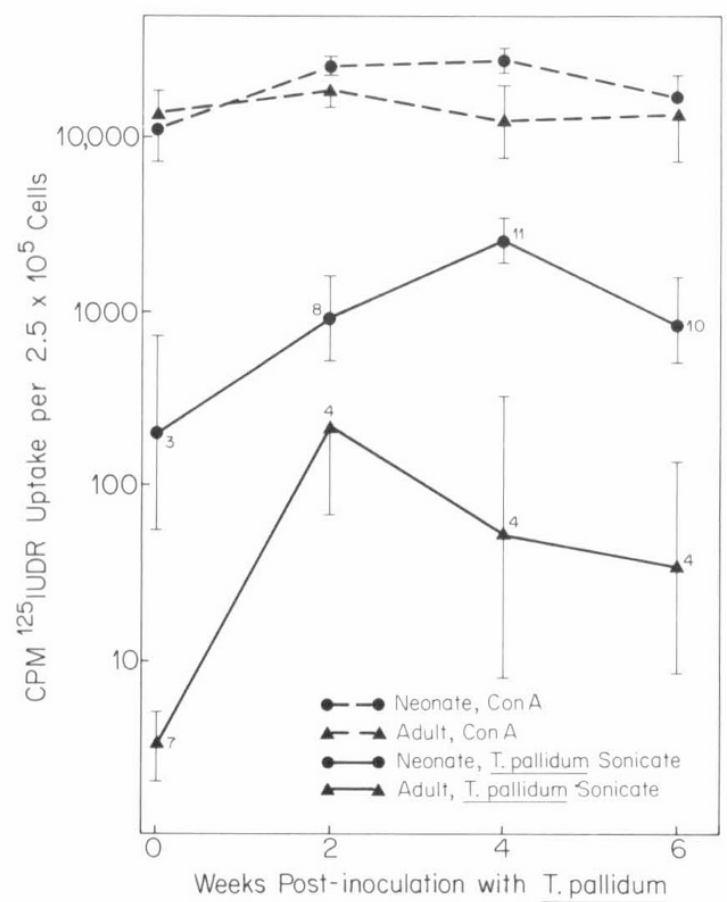

Fig. 2. In vitro proliferative response of popliteal lymph node lymphocytes from neonatal and adult control rabbits to sonicated T. pallidum and Con A after intradermal inoculation with virulent $T$. pallidum. Legend is the same as Figure 1.

point and a maximum of $2580 \mathrm{cpm}$ at 4 weeks postinoculation (Fig. 2). Further evidence for neonatal immunocompetence was provided by lymphocyte responsiveness to Con A which was comparable to that of adult animals (Figs. 1 and 2). 
As shown in Figure 3, splenic lymphocytes from animals inoculated with heat-killed treponemes also responded to $T$. pallidum antigens in the blast transformation assay. These data seem to suggest that responsiveness is not necessarily the result of active infection. However, the responsiveness was substantially lower than that of animals inoculated with virulent $T$. pallidum. Further, the same differences in magnitude of responses were not found between neonate and adult control animals inoculated with virulent $T$. pallidum; neonates inoculated with virulent $T$. pallidum demonstrated responses either similar to (Fig. 1) or greater than (Fig. 2) responses of adult animals who had developed typical, progressive lesions upon similar inoculation and were known to be infected.

Antibody responses. Animals were tested for VDRL and FTAABS antibodies at 1, 2, 4, 6, 8, and 12 weeks postinoculation with virulent $T$. pallidum. As shown in Figure 4, the production of VDRL and FTA-ABS antibody by neonates lagged behind that of adult controls. Two weeks after inoculation, the mean VDRL titer of adult animals reached a maximum of 22.6 dils, then slowly declined over the next 10 -week period to 3.2 dils (Fig. $4 A$ ). In contrast, VDRL antibody among neonates was not detected until 6 weeks postinoculation, at which time a mean titer of 1.2 dils was obtained; during the following 6 weeks, the mean titer increased to 11.6 dils. The FTA-ABS antibody in adult animals increased from a mean titer of 3.9 at 1 week postinoculation to 320.0 at 4 weeks postinoculation (Fig. $4 B$ ). At 6 weeks postinoculation, the mean titer dropped, then increased significantly to 2031.9 and 2560.0 at 8 and 12 weeks postinoculation, respectively. In contrast, FTA-ABS antibody among the neonatal rabbits inoculated with virulent $T$. pallidum was not detectable until 4 weeks postinoculation. At this time, the mean titer was 1.2 and steadily increased to 702.2 at 12 weeks postinoculation. It is important to note that the adult levels of FTA-ABS antibody were significantly greater than those of the neonates throughout the observation period.

Antibody responses to heat-killed T. pallidum were determined in a similar manner. Neonates failed to produce detectable VDRL or FTA-ABS antibody throughout a 6-week period. Only five of 15 adult animals tested within the first 6 weeks after

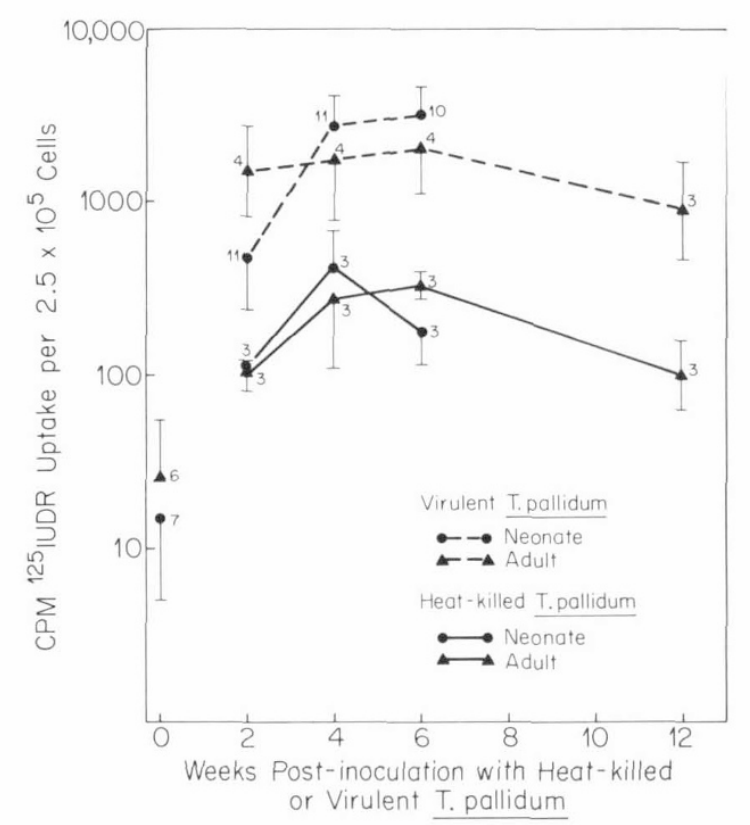

Fig. 3. In vitro proliferative response of splenic lymphocytes from neonatal and adult control rabbits to sonicated $T$. pallidum after intradermal inoculation with $1 \times 10^{6}$ virulent or heat-killed $T$. pallidum at each of two sites. Responses to Con A were of the same magnitude as demonstrated in Figures 1 and 2, and are not shown. Legend is the same as Figure 1.
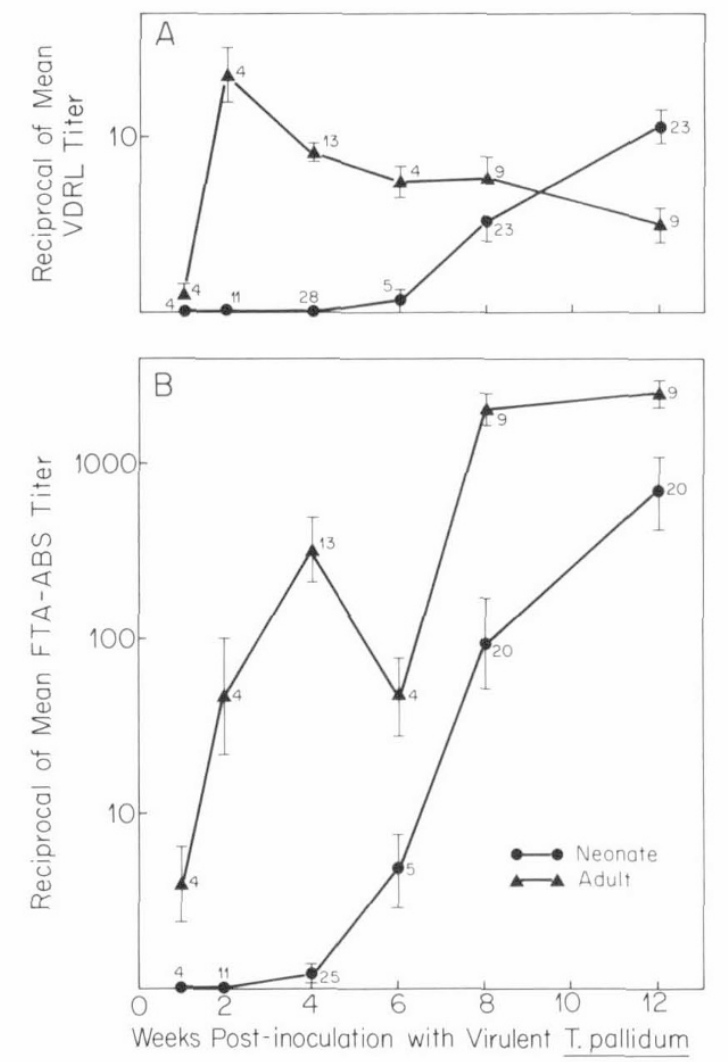

Fig. 4. Serological test results of sera taken from neonatal and adult control rabbits at $1,2,4,6,8,10$, and 12 weeks postinoculation with virulent $T$. pallidum. Neonates were inoculated at 5 to 8 days of age. The number of sera represented at each time point are indicated. The data points are the geometric mean $\pm \mathrm{SE}$ of the reciprocal titers for the indicated number of sera. $A$, reciprocal mean VDRL titers. A 6-week delay in detectable VDRL antibody in sera of neonates is observed. $B$, reciprocal mean FTA-ABS titers. A 4-week delay in detectable FTA-ABS antibody is sera of neonates is observed.

inoculation produced VDRL antibody and were of low titers ranging from 1 to 4 dils, after which no further antibody was detected. An FTA-ABS antibody response was not detected at any of the time points during a 12-week observation period. It was interesting to note that three serum samples obtained from adult animals at 12 weeks after inoculation with heat-killed $T$. pallidum all failed to neutralize virulent organisms by the in vitro-in vivo method of Bishop and Miller (3).

Examination of dermal sites of inoculation by immunofluorescent microscopy. Sections of skin taken from the site of $T$. pallidum-inoculated neonatal rabbits who either failed to develop lesions or developed atypical lesions were prepared for examination by immunofluorescent microscopy. Sections of skin were prepared in a similar manner from the infected adult control animals, each of whom developed typical lesions. The sections were prepared from animals at $1,2,4$, and 6 weeks postinoculation and labeled with the appropriate fluorescein-tagged globulin to identify the presence of T. pallidum, T lymphocytes, or immunoglobulin (B lymphocytes). Neither T. pallidum nor a cellular infiltrate was seen in skin sections from neonates that had remained free of lesions up to the time of sampling. This was in contrast to those findings observed among the les( + ) neonates and adult rabbits who had developed lesions at some time following intradermal inoculation.

One week following inoculation, significant $T$. pallidum were visible in the dermal tissue of les( + ) neonatal (Fig. $5 \mathrm{~A}$ ) and adult (Fig. 5D) animals. At 2 weeks postinoculation, a substantial increase in the number of treponemes was seen among the les $(+)$ neonates (Fig. $5 B$ ). However, the observed increase was consid- 
erably less than that observed among the adult rabbits (Fig. $5 E$ ). Interestingly, the observed increase of treponemes in neonate sections was paralleled by an apparent increase in fragmented organisms (Fig. $5 C$ ). At 4 weeks postinoculation, $T$. pallidum was essentially cleared from both the les(+) neonate and adult dermis. Only an occasional organism was identified at 6 weeks postinoculation and these were in sections from adults (Fig. $5 F$ ).

A mononuclear cell infiltrate was apparent in both neonatal and adult animals. Comparative staining of serial sections with ATS and anti-Ig showed the infiltrate to be composed predominantly of T cells as shown in Figures 6 and 7. Diffuse staining of immunoglobulin was evident (Figs. $6 \mathrm{~B}, 6 \mathrm{D}, 6 \mathrm{~F}, 7 \mathrm{~B}$, and $7 \mathrm{D}$ ); however, B lymphocytes were scattered and rarely seen. A concentration of T lymphocytes was observed surrounding the blood vessels and hair follicles at all time points in both neonates and adult controls. Examples of this perifollicular and perivascular localization is shown in sections taken at 2 and 6 weeks postinocuation (Figs. $6 A, 6 C, 6 E$, and $8 C$ ).

The infiltrate (Figs. $7 A$ and $8 A$ ) had significantly increased in both neonate and adult animals by 2 weeks postinoculation (Figs. $7 C$ and $8 B$ ); immunoglobulin staining remained intense and diffuse (Figs. $6 B, 6 D, 6 F$, and $7 D$ ). At 4 weeks postinoculation, the inflammatory response had begun to subside as evi- denced by the declining numbers of $\mathrm{T}$ lymphocytes among adult animals and the relatively few seen in neonate sections (Fig. $7 E$ ). By 6 weeks postinoculation, the number of $T$ lymphocytes had steadily declined in adult animals; the largest concentration remained perifollicularly (Fig. $8 C$ ). The pattern of immunoglobulin staining followed that of the T lymphocyte infiltrate; as the cell number diminished so did the intensity of the immunoglobulin stain.

Lesions present among neonates at 1 week postinoculation were raised, indurated, and small with diameters ranging from 3 to $6 \mathrm{~mm}$. In contrast, adult lesions were erythematous and larger with diameters ranging from 8 to $14 \mathrm{~mm}$; in addition, they possessed a smooth oozing surface indicative of developing ulceration. At 2 weeks postinoculation, adult lesions were typically ulcerated while neonatal lesions either remained unchanged or had receded. At 4 weeks postinoculation, adult lesions were in the process of healing while the atypical neonatal lesions, which are commonly of short duration (9), had long since receded.

Resistance to challenge. In an effort to demonstrate a refractory state among neonatal rabbits, 23 animals previously inoculated intradermally with $1 \times 10^{6} \mathrm{~T}$. pallidum at each of two sites at age 5 to 9 days were challenged 78 to 152 days later with the same concentration of treponemes by the same route at two to
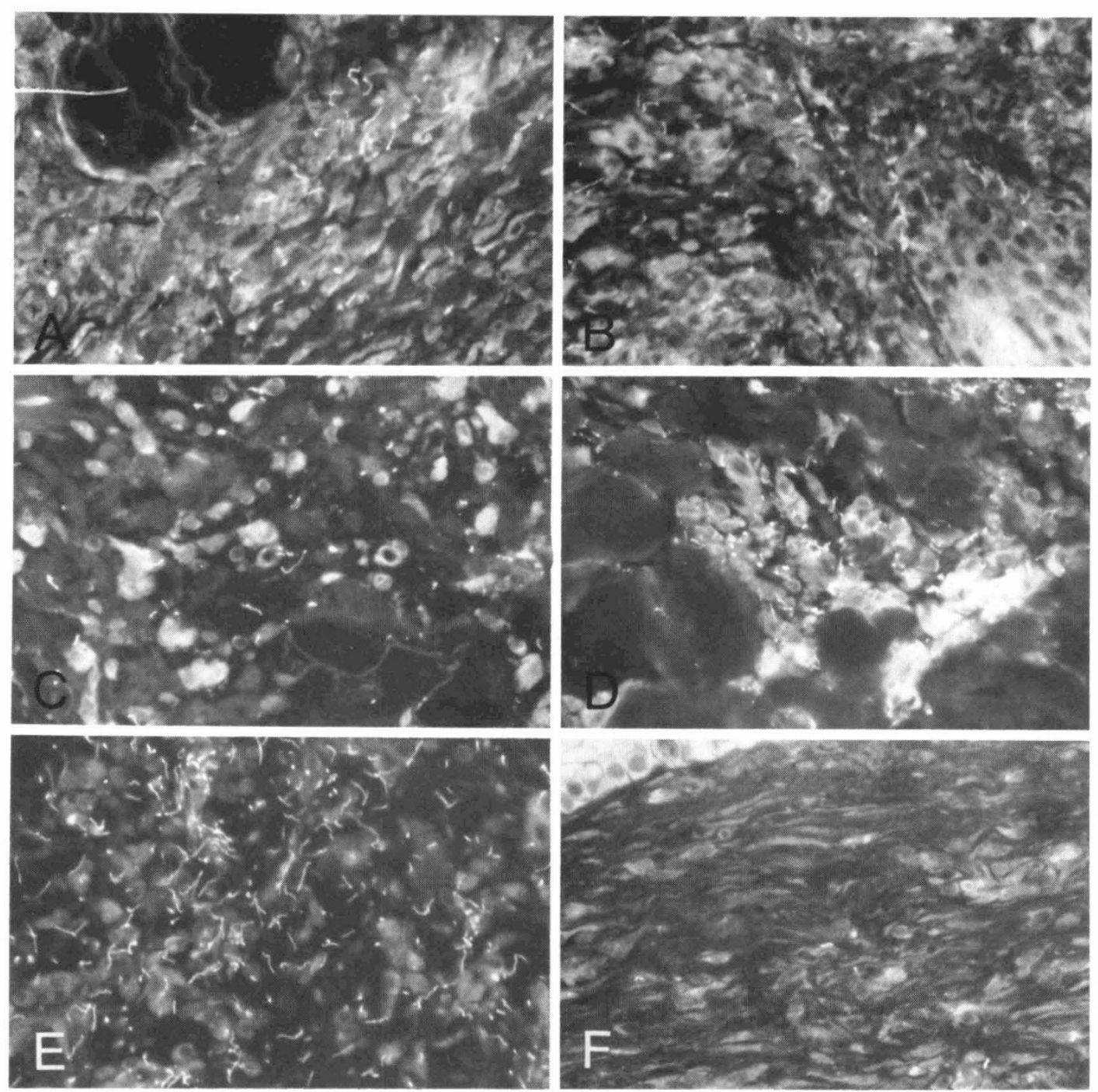

Fig. 5. T. pallidum at the site of ioculation of les $(+)$ neonate and adult control rabbits injected with $1 \times 10^{6}$ virulent $T$. pallidum. T. pallidum $(\times 400)$ in dermal sections from neonates $(A-C)$ and adult control animals $(D-F)$ stained by indirect immunofluorescence. The number of treponemes visible at 1 week postinoculation in both neonate $(A)$ and adult control $(D)$ sections are significantly increased at 2 weeks postinoculation $(B$ and $E$ ) and to a greater degree among adult control animals $(E)$. Paralleling the increase seen in neonates is an increase in fragmented treponemes at 2 weeks postinoculation $(C)$. By 6 weeks postinoculation, rare organisms are visible only in adult sections $(F)$. 

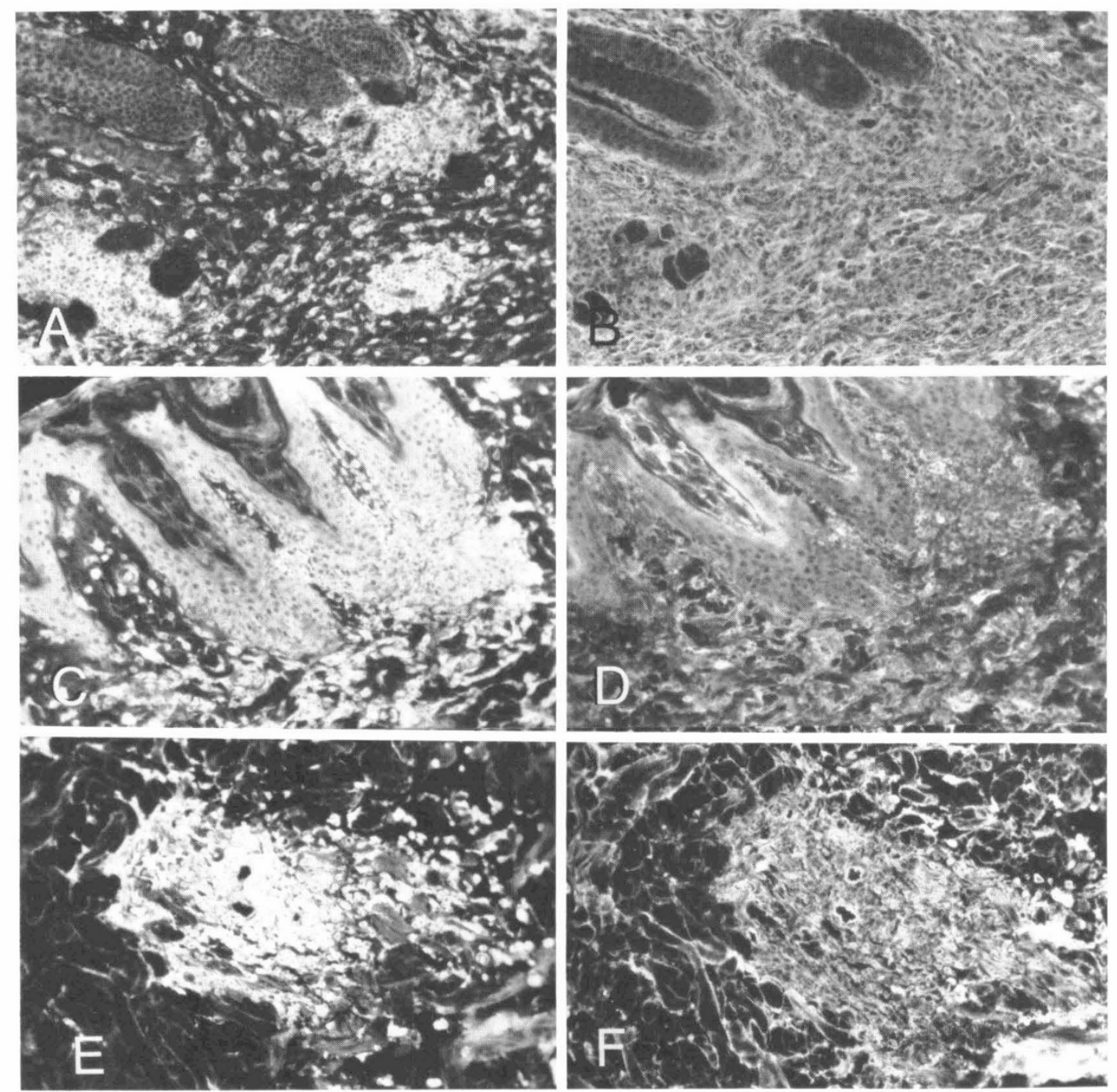

Fig. 6. T cells and immunoglobulin in neonate and adult control serial sections of dermis at 2 weeks postinoculation with virulent $T$. pallidum. The indirect immunofluorescent staining of neonate $(A$ and $B)$ and adult control $(C-F)$ serial sections $(\times 160)$ with ATS $(A, C$, and $E)$ and anti-Ig $(B, D$, and $F)$ demonstrates the $\mathrm{T}$ cell nature of the infiltrate and its perifollicular $(A$ and $C)$ and perivascular $(E)$ concentration.

four sites; adult animals were similarly inoculated with the same suspension. In contrast to the development of typical lesions among the normal adult control animals, 22 of the 23 neonates and all seven infected adult control animals failed to develop any lesions and were therefore considered immune to challenge (Table 1). This was indicative of a generalized asymptomatic infection resulting from the primary inoculation. Uninfected rabbits challenged intradermally at the same age as the maturing neonates in this study and with a similar inoculum failed to exhibit any resistance to $T$. pallidum; typical progressive, ulcerative lesions uniformly developed after the expected incubation period.

Generalized lesion development. Further evidence in support of asymptomatic infection among inoculated neonates was provided by the occurrence of late generalized lesions. Seven of 29 neonates from four experiments developed generalized lesions on the nose, paws, and/or tail, 2 to 3 months after primary inoculation. Dark field microscopic examination of aspirates from representative lesions were positive for $T$. pallidum. Significantly, the lesions were found on neonates irrespective of whether they remained free of or developed atypical lesions on primary inoculation. It was of interest to note that generalized lesions failed to occur among the adult control animals.

\section{DISCUSSION}

Our results do not support the concept expressed by other investigators that depressed cellular responsiveness is induced by
T. pallidum infection (25-27). Indeed, we have shown that cellular immune mechanisms are functional in neonatal and adult rabbits as measured by the transformation of spleen and popliteal lymph node cells in response to both Con A and $T$. pallidum antigens. In fact, the response of lymph node lymphocytes from neonates to $T$. pallidum antigens was greater than that of the same cells from adults; this may be due to differences in lymphocyte homing patterns between the neonate and adult rabbits or to differences in antigen distribution. No attempt was made to purify $\mathrm{T}$ and $\mathrm{B}$ cells from the spleen and popliteal lymph node preparations utilized in this study. However, Lukehart et al. (18) have demonstrated in identically performed assays that the in vitro proliferative response by lymphoid cells from adult rabbits infected with $T$. pallidum is T cell mediated, thus providing compelling evidence that our observed responses are also $\mathrm{T}$ cell mediated. Further, antibody production was delayed in neonatal rabbits, indicating an immature antibody producing apparatus and thus making it unlikely that B cell transformation contributed significantly to the response. Our demonstration of cellular responsiveness by neonatal rabbits to $T$. pallidum was not surprising considering the numerous observations of cellular immunocompetency by neonatal rabbits $(11-13,15,16,24,36)$.

The delay in antibody production to $T$. pallidum by neonatal rabbits observed in this study was in contrast to the earlier appearance of cellular responsiveness among these animals. Delay in antibody production in neonates is a pattern reported for other antigens as well $(2,10,29,30,36,37)$. Sterzl and Trnka 
(37) using inocula of $10^{8}$ heat-inactivated Salmonella paratyphi $B$, detected antibody 20 to 49 days after the injection of 5-dayold rabbits. A delay in antibody response to Salmonella antigens was also reported by Bellanti et al. (2) for animals inoculated at birth. It appears that the ability to respond to antigenic stimulation with the production of specific antibody is not well developed immediately after birth. The data presented in this study show that antibody responsiveness of neonatal rabbits to $T$. pallidum antigens develops by the 2 nd month of life.

An alternative explanation for the delayed antibody production in the presence of positive cellular responses of neonatal rabbits to $T$. pallidum antigens could be a state of split tolerance. However, conditions surrounding tolerance induction and its maintenance make the concept less acceptable. Unlike the ease with which neonatal rabbits can be made tolerant to heterologous serum proteins $(6,30,35,41)$, tolerance induction to bacterial cells has been difficult, a difficulty contributing to the complexity and high antigenicity of the bacterial cell (41). Smith and Bridges (35) were unable to induce tolerance in rabbits inoculated at birth to a variety of bacterial antigens. More relevant was the inability of Festenstein and Bokkenheuser (7) to induce tolerance in neonatal rabbits to $T$. pallidum when inoculated within $5 \mathrm{~h}$ of birth. In addition, the spontaneous termination of a tolerant state has been related to the elimination of the antigen (41); inasmuch as proliferating organisms would be expected to pro- vide a continued source of antigen, it seems unreasonable that a tolerant state would be terminated.

The absence of antibody production by neonates to heat-killed $T$. pallidum was not surprising considering the shortness of the 6 -week observation period and the pattern of delay in response to virulent organisms. High titer nontreponemal antibody by adult rabbits has been reported only after repeated inoculations with large doses of inactivated $T$. pallidum $(5,22)$. Therefore, the low titer VDRL antibody produced by one-third of the inoculated adults and the absence of specific FTA-ABS antibody to heat-killed treponemes in this study was not unexpected. However, the finding that heat-killed T. pallidum failed to stimulate serum neutralizing activity in the three animals tested was of considerable interest inasmuch as such activity has been shown to be associated with acquired resistance (3) and immunity to challenge does not develop in animals inoculated with heat-killed organisms (5).

Spleen cells from both neonate and adult rabbits inoculated with heat-killed $T$. pallidum responded to $T$. pallidum antigens in a blast transformation assay to a substantially greater extent than uninoculated animals. Although the kinetics of the responses were similar to those seen among animals inoculated with virulent $T$. pallidum, the magnitude of the resonse was less by approximately one log. In contrast, Pavia et al. $(25,26)$ found peripheral blood lymphocytes from rabbits inoculated with heat-
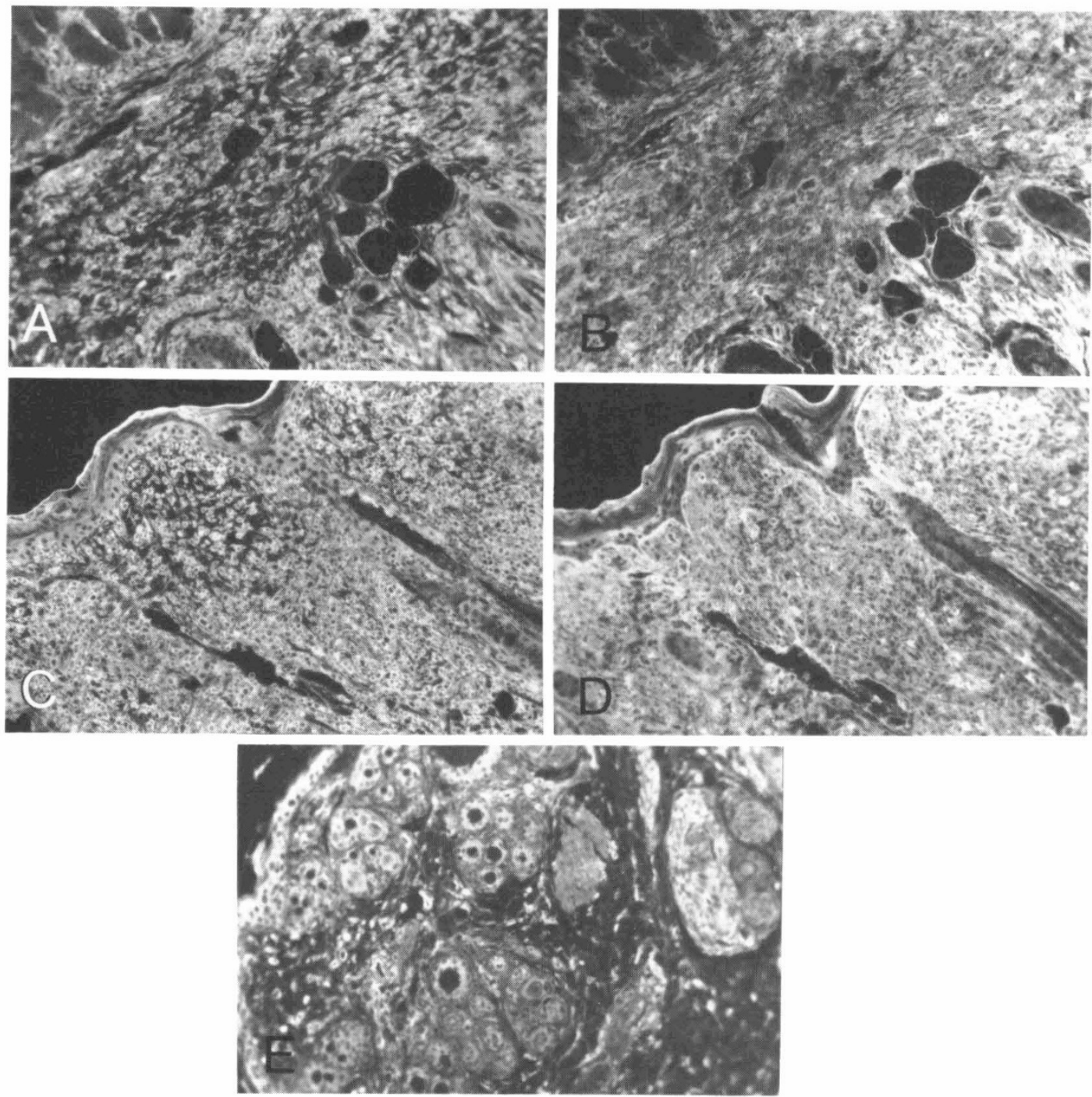

Fig. 7. T cells and immunoglobulin in neonate dermal sections at 1,2 , and 4 weeks postinoculation with virulent $T$. pallidum. $A, B, C$, and $D$ $(\times 160)$ :serial sections stained by ATS $(A$ and $C)$ and anti-Ig $(B$ and $D)$ show a T cell infiltrate and an absence of B cells in the presence of immunoglobulin. The infiltrate at 1 week postinoculation $(A)$ is increased at 2 weeks postinoculation $(C)$ and minimal at 4 weeks postinoculation $(E)$. 

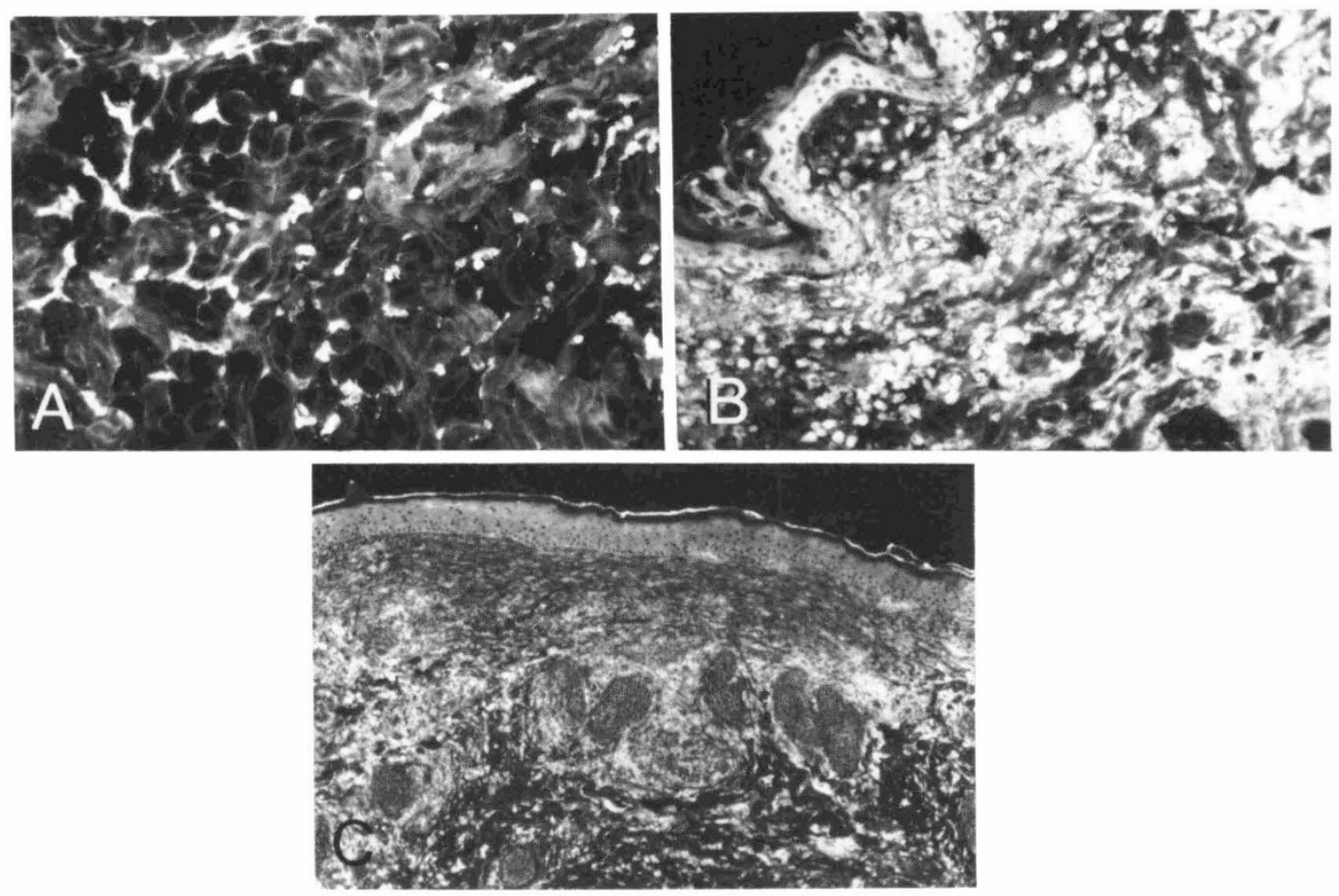

Fig. 8. T cells in dermal sections from adult control animals at 1,2 , and 6 weeks postinoculation with virulent $T$. pallidum $(\times 160)$. The $T$ cell infiltrate of 1 week postinoculation $(A)$ increases and maximizes at 2 weeks postinoculatin $(B)$. By 6 weeks postinoculation, the infiltrate is greatly diminished and is concentrated largely perifollicularly $(C)$.

Table 1. Resistance of neonates previously inoculated with $T$. pallidum to homologous challenge

\begin{tabular}{|c|c|c|c|}
\hline \multirow[b]{2}{*}{ Animals } & \multirow[b]{2}{*}{$\begin{array}{l}\text { Number of } \\
\text { animals }\end{array}$} & \multicolumn{2}{|c|}{ Lesion pattern* } \\
\hline & & $\begin{array}{c}\text { Primary } \\
\text { inoculation } \dagger\end{array}$ & $\begin{array}{c}\text { Challenge } \\
\text { inoculation }\end{array}$ \\
\hline \multirow[t]{3}{*}{ Neonates } & 13 & $\begin{array}{l}+ \\
\mathrm{cal}) \S\end{array}$ & - \\
\hline & 9 & - & - \\
\hline & 1 & - & + (typical) $)^{q}$ \\
\hline \multirow[t]{2}{*}{ Adult controls } & 7 & + (typical) & - \\
\hline & 2 & Not done & + (typical) \\
\hline
\end{tabular}

$*+$ lesions; - , absence of lesions.

$\dagger$ Intradermal inoculation of neonates 5 to 9 days of age at two sites and adult control at two or four sites with $1 \times 10^{6} \mathrm{~T}$. pallidum $/$ site.

$\ddagger$ Intradermal inoculation of animals 78 to 152 days after primary inoculation at two to four sites with $1 \times 10^{6} \mathrm{~T}$. pallidum/site.

$\S$ Atypical, indurated, small, nonulcerative, nonprogressive lesions that appeared after varied incubation periods (see Ref. 9).

I Typical, erythematous, indurated, enlarged lesions that appeared after the appropriate incubation period and progressed to ulceration.

killed $T$. pallidum unresponsive compared to pre-inoculation levels. The discrepancy in data may reside in the lymphocyte source; peripheral blood lymphocytes from infected adult animals have been shown to give variable results in response to $T$. pallidum antigens (1). Thus, the transformation assay, as performed in our laboratory on spleen cells from animals inoculated with virulent $T$. pallidum cannot be used as definitive evidence of asymptomatic infection. However, differences in magnitude of responses may reflect differences in degrees of sensitization of the host. The expected response from a host exposed to persistent stimulation and increasing antigenic load provided by proliferating organisms should be greater than the host response to dead organisms.

The local cellular reactions among neonates with atypical lesions differed from those of adult animals only in intensity and thus provided additional evidence for cellular responsiveness. The absence of B cells was not surprising considering reports of an early predominantly $\mathrm{T}$ cell infiltrate response (17) and $\mathrm{T}$ cell hyperplasia of lymphoid tissue (31) among adult rabbits infected with $T$. pallidum by the intratesticular route. Thus, the cellular response of neonates follows a pattern of events similar to that of adults yet without the appearance of typical lesions. The primary cutaneous lesion in the adult rabbit is caused by a delayed hypersensitivity skin reaction. The fact that resistance to reinfection does not occur until several months following the manifestation of the primary reaction suggests that prolonged survival is required for development of resistance in the adult. The absence of cutaneous lesions upon primary inoculation of neonatal rabbits and the development of resistance to challenge suggests full development of cellular hypersensitivity may be delayed. Full development of the primary delayed hypersensitivity response may be impaired because the innate resistance demonstrated is effective in preventing an increase in the number of organisms required to elicit the primary skin reaction. However, dissemination of some organisms is sufficient to induce a later state of acquired immunity. Examination of the sites of inoculation for treponemes revealed either their absence or considerably fewer organisms among neonates as compared to adult animals inoculated with the same suspension. In addition, the observed increase in fragmented organisms in neonate sections supports the contention that the number of organisms is more rapidly reduced.

The early absence of antibody among neonates and the similar cellular responses evoked by both neonates and susceptible adults in this study tend to preclude the concept that either humoral or cellular immune mechanisms are involved in neonate resistance. Though it is possible that more sensitive methods might reveal such a relationship, it apears more likely that neonatal resistance may be related, at least in part, to nonimmune factors (9).

We have clearly demonstrated the innate resistance of neonatal rabbits to symptomatic infection with $T$. pallidum; the question of whether the organisms were completely eliminated or persisted as an asymptomatic infection was not determined (9). In the 
present study, treponemes could be found after 2 weeks at the inoculation sites of $T$. pallidum-injected neonates who had developed atypical lesions. Our challenge data have provided evidence for the persistence of treponemes among both les(+) and les(-) neonates at least 3 months after primary inoculation. Twenty-two of the 23 neonates challenged with large numbers of $T$. pallidum approximately 3 to 5 months after primary inoculation were refractive to challenge. In adult rabbits, this refractive or immune state is evident as early as 11 days and complete by 3 months after primary inoculation (3). An early elimination of organisms precludes the development of acquired resistance in adult animals $(14,19,20,40)$. Inasmuch as adult rabbits are incapable of eliminating $T$. pallidum without curative theray $(19,39,40)$, it is reasonable to assume that 3 -month-old neonates are also incapable of eliminating the organism and therefore most likely harbor an asymptomatic infection. Thus, the immunity to challenge demonstrated by neonates in this study was indicative of treponemal persistence. The late development of generalized lesions among some neonates provided additional evdence for the occurrence of earlier asymptomatic infection.

It has generally been accepted that the human fetus is immunologically disadvantaged and highly susceptible to $T$. pallidum infection. However, careful examination of the literature does not entirely support this concept. Silverstein et al. $(33,34)$ and Fogel et al. (8) have shown that the human fetus does in fact respond immunologically to $T$. pallidum infection. Further, the concept of fetal susceptibility has been based primarily upon the overt clinical manifestations of early congenital infections presented at birth or shortly thereafter. However, late congenital syphilis, in which $60 \%$ of the patients are latent (38), may represent a status of resistance similar to that observed in the rabbit. If these and other questions relating to the pathogenesis and immunology of this form of the disease are to be answered, the development of an experimental congenital and neonatal syphilis model is essential.

Acknowledgments. The authors are indebted to R. M. Cheri Lloyd and Roxanne Holdbrook for invaluable technical assistance, to Judy G. Wong for graphics, and to Emma Hollins for the careful typing of the manuscript.

\section{REFERENCES}

1. Baker-Zander SA, Sell S 1980 A histopathologic and immunologic study of the course of syphilis in the experimentally infected rabbit. Demonstration of a long-lasting cellular immunity. Am J Pathol 101:387

2. Bellanti JA, Eitzman DV, Robbins JB, Smith RT 1963 The development of the immune response. Studies on the agglutinin response to Salmonella flagellar antigens in the newborn rabbit. J Exp Med 117:479

3. Bishop NH, Miller JN 1976 Humoral immunity in experimental syphilis. II. The relationship of neutralizing factors in immune serum to acquired resistance. J Immunol 117:197

4. Chesney A 1926 Immunity in syphilis. Medicine 5:463

5. Eagle H. Fleischman R 1948 The antibody response in rabbits to killed suspensions of pathogenic T. pallidum. J Exp Med 87:369

6. Eitzman DV, Smith RT 1959 Antibody response to heterologous protein in rabbits of varying maturity. Proc Soc Exp Biol Med 102:529

7. Festenstein H, Bokkenheuser V 1961 Attempted induction of immunological tolerance in rabbits using living Treponema pallidum. $\mathrm{Br} \mathrm{J}$ Exp Pathol 42:158

8. Fogel VJ, Tamer AM. Fojaco RM, Herrera JA, Frank P 1969 Immunologic response to the fetus in congenital syphilis. J Florida Med Assoc 56:777

9. Gamboa D, Miller JN 1984 Experimental neonatal syphilis. I. Evidence of resistance to symptomatic infection in neonatal rabbits following intraderma inoculation with Treponema pallidum (Nichols strain). Pediatr Res 18:965

10. Harris S, Harris TN, Ogburn CA, Farber MB 1962 Neonatal rabbits as donors of transferred lymph node cells. J Immunol 88:206

11. Harris TN, Harris S, Farber MB 1962 Transfer of rabbit lymph node cells to neonatal recipient rabbits. J Immunol 88:199

12. Harris TN, Harris S. Farber MB 1959 Effects of injection of rabbit leucocytes into neonatal rabbits on subsequent lymph node transfer. Proc Soc Exp Biol Med 102:495

13. Harris TN, Harris S, Farber MB 1962 Immunologic response of neonatal and older rabbits to antigens of rabbit leucocytes. J Exp Med 116:575

14. Hollander DH, Turner TB, Nell EE 1952 The effect of long continued subcurative doses of penicillin during the incubation period of experimental syphilis. Bull Johns Hopkins Hosp 90:102

15. Holub M 1962 The fate and morphological differentiation of lymphoid cells transferred to young rabbits. Transplant Bull 5:73

16. Knight S, Ling NR 1969 A comparison of the responses of lymphocytes from blood, spleen, thymus and appendix of rabbits of different ages to stimulation in vitro with staphylococcal filtrate. Clin Exp Immunol 4:667

17. Lukehart SA, Baker-Zander SA, Lloyd RMC, Sell S 1980 Characterization of lymphocyte responsiveness in early experimental syphilis. II. Nature of cellular infiltration and Treponema pallidum distribution in testicular lesions. J Immunol 124:461

18. Lukehart SA, Baker-Zander SA, Sell S 1980 Characterization of lymphocyte responsiveness in early experimental syphilis. I. In vitro response to mitogens and Treponema pallidum antigens. J Immunol 124:454

19. Magnuson HJ, Rosenau BJ 1948 the rate of development and degree of acquired immunity in experimental syphilis. Am J Syph 32:418

20. Magnuson HJ, Thompson FA Jr, Rosenau BJ 1952 The effect of subcurative doses of penicillin during the incubation period of experimental syphilis. Bull Johns Hopkins Hosp 90:105

21. Manual of Tests for Syphilis 1964, 1969 United States Department of Health Education, and Welfare Publication 411. Public Health Service, National Communicable Disease Center, Atlanta

22. McLeod CP, Magnuson HJ 1953 Production of immobilizing antibodies unaccompanied by active immunity to Treponema pallidum as shown by injecting rabbits and mice with killed organisms. Am J Syph 37:9

23. Miller JN, Whang SJ, Fazzan FP 1963 Studies on immunity in experimenta syphilis. I. Immunologic response to rabbits immunized with Reiter protein antigen and challenged with virulent Treponema pallidum. $\mathrm{Br} \mathrm{J}$ Vener Dis 39:195

24. Najarian JS, Dixon FJ 1962 Homotransplantation immunity of neonatal rabbits. Proc Soc Exp Biol Med 109:592

25. Pavia CS, Baseman JB, Fold JD 1962 Selective response of lymphocytes from Treponema pallidum-infected rabbits to mitogens and Treponema reiteri. Infect Immun 15:417

26. Pavia CS. Folds JD, Baseman JB 1976 Depression of lymphocyte response to concanavalin $\mathrm{A}$ in rabbits infected with Treponema pallidum (Nichols strain). Infect Immun 14:320

27. Pavia CS, Folds JD, Baseman JB 1977 Selective in vitro response of thymusderived lymphocytes from Treponema pallidum-infected rabbits. Infect Immun 18:603

28. Pepose JS, Bishop NH. Feigenbaum S, Miller JN, Zelter PM 1980 The humoral immune response in rabbits infected with Treponema pallidum: comparison of antibody levels measured by the staphylococcal protein A-IgG (SPA-TP) microassay with VDRL, FTA-ABS, and TPI antibody responses during the development of acquired resistance to challenge. Sex Transmitted Dis 7:125

29. Pernis B, Cohen MW. Thornbecke GJ 1963 Specificity of reaction to antigenic stimulation in lymph nodes of immature rabbits. I. Morphologic changes and $\gamma$-globulin production following stimulation with diphtheria toxoid and silica. J Immunol 91:541

30. Riha I 1963 Antibody formation in young rabbits immunized with heterologous serum proteins. Folia Microbiol 8:17

31. Sell S, Baker-Zander SA, Lloyd RMC 1980 T cell hyperplasia of lymphoid tissues of rabbits infected with Treponema pallidum: evidence for a vigorous immune response. Sex Transmitted Dis 7:74

32. Sell S, Gamboa D, Baker-Zander SA, Lukehart SA, Miller JN 1980 Host response to Treponema pallidum in intradermally infected rabbits: evidence for persistence of infection at local and distant sites. J Invest Dermatol 75:470

33. Silverstein AM 1962 Congenital syphilis and the timing of immunogenesis in the human foetus. Nature 194:196

34. Silverstein AM, Lukes RJ 1962 Fetal response to antigenic stimulus. I. Plasmacellular and lymphoid reactions in the human fetus to intrauterine infection. Lab Invest 11:918

35. Smith RT, Bridges RA 1958 Immunological unresponsiveness in rabbits produced by neonatal injection of defined antigens. J Exp Med 108:227

36. Sterzl J 1958 The determination of the period of metabolic activity of normal spleen cells and those stimulated with antigen, after the transfer into young animals. Transplant Bull 5:73

37. Sterzl J, Trnka Z 1957 Effect of very large doses of bacterial antigen on antibody production in newborn rabbits. Nature 179:918

38. Syphilis, a Synopsis 1968 Publication 1660. United States Public Health Service, Washington, DC, pp 86-95

39. Turner TB, Hollander DH 1957 Immunity phenomena in the treponematoses. In: Biology of the Treponematoses. World Health Organization, Geneva, pp $123-168$

40. Turner TB, Nelson RA Jr 1950 The relationship of treponemal immunobilization antibody to immunity in syphilis. Trans Assoc Am Phys 63:112

41. Weigle WO 1973 Immunological unresponsiveness. Adv Immunol 16:61 